$>$ Le récent développement de la synthèse de protéines membranaires par des systèmes dits acellulaires et la reconstitution des membranes lipidiques planes ont permis l'émergence d'une technologie transversale pour la production de biocapteurs ou de filtres. Un certain nombre de membranes biomimétiques sont actuellement normalisées et utilisées par l'industrie (comme par exemple, un filtre contenant l'aquaporine pour la désalinisation de l'eau), ou en routine à l'échelle du laboratoire (comme la bactériorhodopsine en tant que capteur de lumière). Dans le domaine médical, plusieurs champs d'applications pour ces membranes biomimétiques sont à l'étude pour du criblage de molécules thérapeutiques et pour répondre concrètement aux besoins de développement de nouveaux outils pour le diagnostic, le suivi du patient et la médecine personnalisée. <

Dans leur environnement, les cellules eucaryotes et procaryotes sont soumises à des modifications biologiques et/ou chimiques permanentes. La membrane lipidique est une frontière physique qui permet de temporiser l'impact des variations biochimiques du milieu externe sur le milieu intracellulaire. Les phénomènes d'homéostasie tendent dans un second temps à répondre à ces variations. Les protéines membranaires ont pour fonction de répondre à ces stimulus par le transport de métabolites et/ou par le déclenchement d'une cascade de régulations impliquant le métabolisme interne de la cellule. L'utilisation par les biotechnologies de cette capacité à répondre - et surtout à «sentir »- l'environnement est un défi dont l'aboutissement est l'élaboration de capteurs pour de nouvelles molécules, plus résolutifs, ou plus aisés d'utilisation, voire implantables.

Le domaine des biocapteurs a été stimulé par les progrès dans les méthodes de purification des protéines et par le succès des biocapteurs de glucose utilisés pour le

\section{Les capteurs biomimétiques en recherche biomédicale}

Landry Gayet, Jean-Luc Lenormand

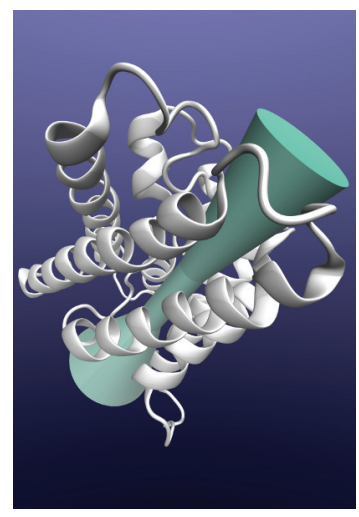

Laboratoire TIMC IMAG, UMR5525, équipe TheREx, CNRS/université Joseph Fourier, bâtiment Jean Roget, domaine de la Merci, 38700 La Tronche, France. landry.gayet@imag.fr jllenormand@chu-grenoble.fr

diagnostic et le suivi du diabète. Cela se traduit par plusieurs milliers d'articles scientifiques chaque année [1]. Dans cette revue, l'accent sera mis sur les biocapteurs utilisant les protéines membranaires, puisque celles-ci représentent des cibles de choix pour l'industrie pharmaceutique (plus de $50 \%$ des médicaments actuels [2]). La capacité à étudier les fonctions de ces protéines a, de ce fait, un intérêt fondamental pour le criblage de molécules ayant un potentiel thérapeutique. L'utilisation de ces protéines membranaires se heurte cependant à deux facteurs limitants. Le premier est la difficulté de leur expression (recombinante) et de leur purification (recombinante et native). Le second est le défi de recréer un microenvironnement membranaire fonctionnel mimant la bicouche lipidique dans laquelle elles sont enchâssées naturellement (membrane biomimétique). Les progrès réalisés dans le domaine de l'expression et de la purification des protéines membranaires, ainsi que les avancées dans le domaine de la génération des membranes biomimétiques, offrent, par leur combinaison, une vaste gamme d'applications.

\section{L'expression et la purification des protéines membranaires}

\section{Production des protéines au sein d'un hôte}

La purification des protéines membranaires est un défi majeur pour la biotechnologie puisque 20 à $30 \%$ des gènes codent pour ces protéines [3]. La production au sein d'un hôte (cellules eucaryotes ou procaryotes) de ces protéines sous forme recombinante, suivie de leur extraction à partir des membranes biologiques est une option qui nécessite un bon niveau d'expression de ces protéines. Cependant, le rendement de cette méthode est souvent limité par l'effet toxique de ces protéines pour l'hôte. De plus, ces protéines étant insolubles, il est nécessaire ensuite de cribler les détergents adaptés à chaque protéine membranaire. 


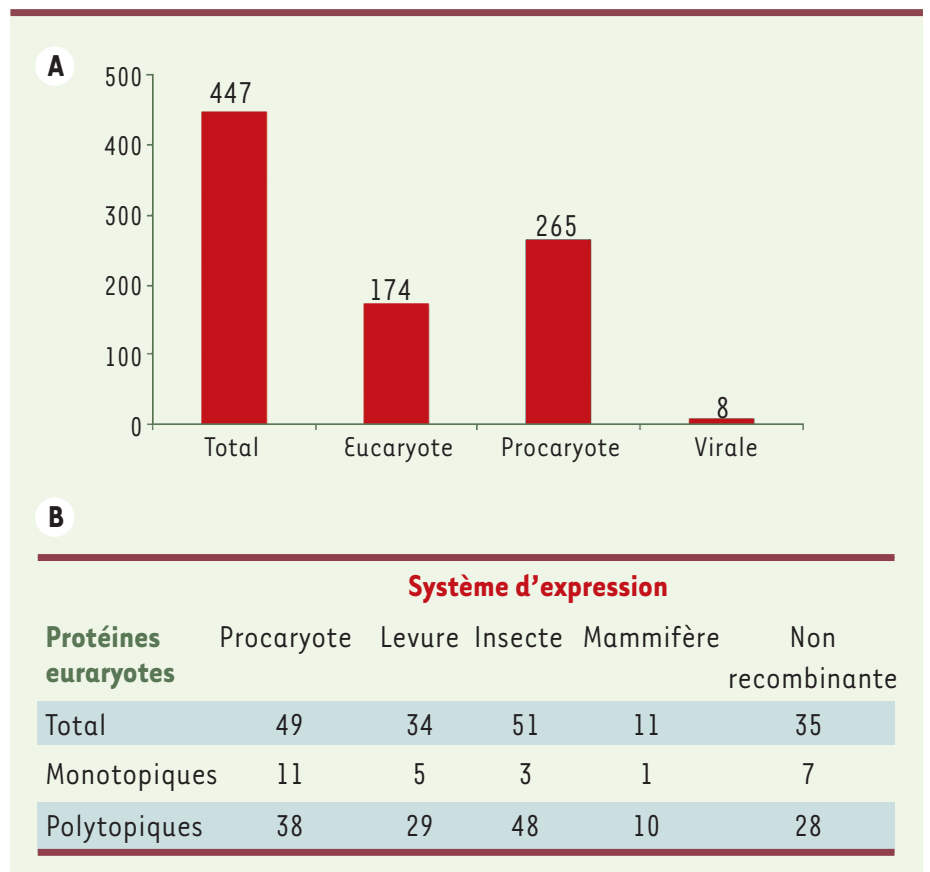

Figure 1. Répartition des protéines membranaires de structure connue. A. Selon leur origine eucaryote, procaryote ou virale. B. Selon le système d'expression utilisé.

Comme pour les protéines solubles, la production de protéines membranaires en Escherichia coli reste la plus largement utilisée [4]. Néanmoins, ce modèle n'est pas forcément le plus approprié pour la production de protéines membranaires d'origine eucaryote. En effet, pour ces protéines, la quantité obtenue et la qualité du produit final (absence de modifications post-traductionnelles, comme par exemple les glycosylations) sont souvent médiocres. Or, ces facteurs sont déterminants pour pouvoir ensuite étudier la structure tridimensionnelle ou cristallographique de ces protéines. La faible expression de ces protéines peut s'expliquer notamment par un effet toxique de la protéine membranaire surexprimée, qui déstabilise les membranes biologiques et l'homéostasie de l'organisme hôte. La mise en place d'un protocole de purification des protéines permettant l'obtention d'une quantité suffisante et d'une pureté significative est donc essentielle pour la détermination structurelle des protéines membranaires. Le site de l'équipe de White (http://blanco.biomol.uci.edu/mpstruc/) répertorie les protéines membranaires dont la structure a été déterminée. Une synthèse des modes de production par catégories de protéines nous permet d'avoir une vision plus précise. Près de 447 structures de protéines membranaires produites de différentes façons ont été identifiées. Parmi celles-ci, $59 \%$ sont d'origine procaryote, $39 \%$ d'origine eucaryote et $2 \%$ d'origine virale (Figure $1 \mathrm{~A}$ ). Près de $90 \%$ des protéines membranaires procaryotes sont produites en système procaryote, la plupart des autres étant des protéines natives directement extraites de la bactérie. Concernant les protéines membranaires eucaryotes, on distingue deux catégories: les protéines polytopiques (protéines en contact avec les deux compartiments, intra- et extracellulaire, séparés par la membrane grâce à une structure en hélices $\alpha$ et tonneaux $\beta$ ) et les monotopiques (en contact avec un seul compartiment). Cette distinction est importante puisque les mécanismes d'insertion des protéines dans les membranes se sont perfectionnés au cours de l'évolution et sont donc différents d'un organisme à un autre. Toutefois, une protéine transmembranaire, notamment en hélices $\alpha$ [5], est plus affectée par cette différence de mode d'insertion qu'une protéine non transmembranaire (monotopique) dont les domaines hydrophobes sont plus limités. Ainsi, pour les protéines polytopiques, la disparité des systèmes de production qui permettent d'obtenir des protéines fonctionnelles est plus importante que pour les protéines monotopiques, avec $26 \%$ des protéines produites en procaryote ( $\varepsilon$. coli), $19 \%$ en levure, $32 \%$ en cellules d'insectes, $7 \%$ en cellules de mammifères (Figure 1B), les protéines restantes (16\%) étant obtenues par extraction directe à partir de l'organisme. Dans le cas des protéines membranaires monotopiques, $44 \%$ sont produites en système procaryote (Figure lB). Le type d'insertion des protéines eucaryotes dans la membrane est donc un indice à prendre en compte dans le choix de l'hôte à tester en priorité. Quoi qu'il en soit, la production des protéines membranaires recombinantes représente toujours une vraie difficulté dans la gestion des risques des projets de bioproduction, puisque l'on devra tester différents hôtes sans certitude de succès. Malgré l'intérêt économique et scientifique que représentent les protéines membranaires, malgré les investissements institutionnels et privés, le nombre réduit de protéines membranaires produites et purifiées en quantités suffisantes pour pouvoir étudier leur structure traduit l'existence d'un verrou technologique dû à la toxicité de ces protéines pour leurs hôtes, et également à la nature hydrophobe de ces protéines, qui favorise la formation de corps insolubles.

C'est pour cette raison que certains laboratoires tentent de développer des hôtes plus adaptés. Par exemple, pour $\varepsilon$. coli, des souches telles que C41, C43 ou Origami résistent mieux à la toxicité des protéines membranaires [6] ou sont capables de réaliser une partie de la glycosylation ( $\varepsilon$. coli MC4100) [7]. D'autres laboratoires ont développé des étiquettes peptidiques favorisant l'agrégation ou l'insertion des protéines dans les membranes, telles que Zera ou Mistic $[8,9]$. Mais ces solutions ne permettent pas actuellement d'entrevoir le début d'une normalisation de la production des protéines membranaires, car leur toxicité est multifactorielle (problème d'insertion dans les membranes, de 
A Production en phase aqueuse B Production en détergent



C Production en membrane reconstituée (exemple : liposome)

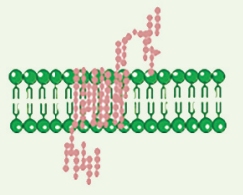

Figure 2. Modes de production en système acellulaire. A. Précipitât. B. Protéine solubilisée en micelles de détergents. C. Protéine insérée dans des membranes artificielles (liposomes, nanodisques). chaperons de repliement, de glycosylation, de composition lipidique des membranes, etc.).

\section{Production acellulaire des protéines}

Outre le système de production cellulaire, depuis les années 1990, la production en milieu acellulaire s'est développée pour les protéines solubles. Aujourd'hui, ce type de production est utilisé dans l'industrie (société Sutro Biopharma). Ce système permet de s'affranchir de I'utilisation d'un hôte devant intégrer la protéine puisque le système de transcription et de traduction ( $\varepsilon$. coli, germe de blé) est produit en amont pour être utilisé in vitro. Certains laboratoires cherchant à produire les protéines membranaires utilisent le système acellulaire de production, car il a l'avantage d'éliminer le problème de la toxicité (Figure 2). D’autre part, la production acellulaire est réalisée en système ouvert et permet donc de faire varier différents facteurs pour optimiser la production des protéines cibles. II s'agit donc d'un système permettant un criblage de conditions beaucoup plus large que le système cellulaire, et il est possible de l'utiliser de manière rentable même sur de petits volumes [10]. Nous avons donc là une réelle solution permettant de répondre au besoin de normalisation de la production des protéines membranaires.

Grâce à la technique acellulaire, l'accès au milieu réactionnel permet d'adapter l'environnement hydrophobe à chaque protéine, ce qui est primordial dans le cas des protéines membranaires. Trois options ont ainsi été développées. La première est la production en milieu aqueux avec solubilisation ultérieure par un détergent, qui rappelle la méthode d'extraction à partir de membranes biologiques (Figure 2A). Cette méthode a rencontré quelques succès avec des protéines telle que hVDACl (voltage dependent anion channel, transporteur de la membrane mitochondriale), obtenue sous forme précipitée puis solubilisée en $\beta$-DDM (dodécyl $\beta$-D maltoside), permettant une production suffisante pour une analyse structurale [11]. La seconde option est la production en présence de détergents (Figure 2B). L'article de Klammt et al. [12] fait un descriptif de cette option pour trois protéines membranaires très différentes structurellement, $\varepsilon m r \varepsilon$ (multidrug transporter composé d'hélices $\alpha$ ), Tsx (nucleoside transporter en tonneaux $\beta$ ) et le récepteur de la vasopressine de la famille des GPCR (G-protein coupled receptor). De façon similaire à l'extraction des protéines membranaires de leur environnement cellulaire naturel, ou des membranes où elles sont surexprimées sous forme recombinante, la production acellulaire nécessite l'utilisation de détergents tels que les alkyles-glucosides, les dérivés des stéroïdes, certains phosphoglycérols ou phosphocholines, et les dérivés des polyéthylène glycols. Par exemple, le Brij-58 (dérivé de polyéthylène glycol) est favorable à la production de Tsx et du récepteur de la vasopressine, mais inhibe totalement la production de $\varepsilon m R \varepsilon$. Cette méthode de production en présence de détergent a notamment permis la production (en présence de digitonine) de la rhodopsine (eucaryote), issue d'une algue marine, en quantité suffisante pour une analyse structurale [13]. La troisième option est la production directe en membrane artificielle (Figure 2C), qui permet la production de protéines membranaires par insertion directe dans des liposomes composés des lipides naturels (par exemple provenant des thylacoïdes de feuilles d'épinards) [14, 15]. Ce schéma de production a l'avantage d'être celui qui correspond le mieux à ce qui se passe in vivo puisque le repliement de la protéine membranaire se fait dans une bicouche lipidique. De plus, ce mode de production permet de passer directement de la production au contrôle d'activité puisqu'il n'est pas nécessaire de réintégrer la protéine produite dans une membrane biomimétique. D’autre part, l'étude de Liguori et al. [14] montre que deux protéines, hBak et hVDACl, peuvent être produites et purifiées simultanément dans le même liposome, ce qui offre de nouvelles options technologiques pour l'étude de partenaires protéiques connus pour fonctionner en coopération.

\section{Les membranes biomimétiques}

Les membranes biomimétiques planes ont été développées depuis les années 1980. Les méthodes de production de membranes planes consistent soit à utiliser la technique de Langmuir-Blodgett par transfert de monocouches de lipides sur un support solide (Figure 3A), soit à réaliser la fusion de liposomes sur un support solide pour former une bicouche de lipides (Figure 3B) [16]. 


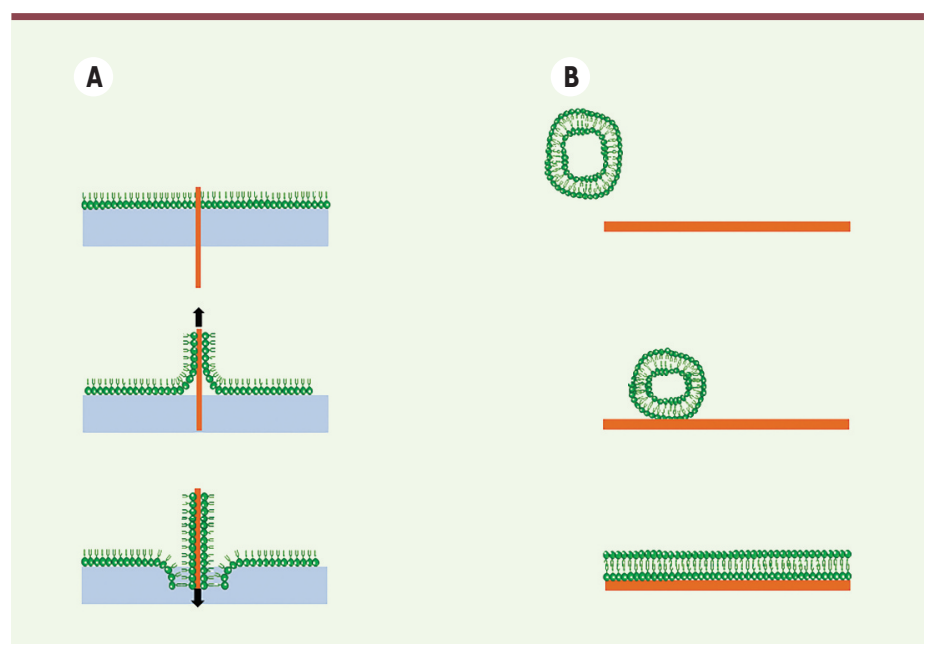

Figure 3. Formation des bicouches lipidiques sur support solide. A. Par la technique de Langmuir-Blodgett. B. Par fusion de liposomes.

Cette deuxième méthode est particulièrement adaptée puisqu'il existe une méthode acellulaire permettant la production de protéines membranaires directement en liposomes. Elle offre en outre la possibilité d'utiliser des liposomes ayant intégré plusieurs types de protéines. Il existe trois architectures majeures de membranes planes. Chacune présente des intérêts différents et trouve des applications en biotechnologies.

L'architecture de base (Figure $4 A$ gauche) est constituée d'un support solide sur lequel est déposée une bicouche lipidique. Ce format est stable, mais ne permet pas le mouvement libre des lipides qui sont contraints par le frottement sur le support solide. Ce format ne permet pas non plus l'intégration des protéines transmembranaires qui ne trouvent pas l'espace nécessaire à leur repliement entre le support et la bicouche. Néanmoins, cette construction a pu être utilisée pour mesurer les interactions directes lipide-protéine par résonance plasmonique de surface (SPR) [17] (Figure 4A centre). Cette architecture permet également l'ancrage d'anticorps couplés à la biotine pour l'obtention de membranes «fonctionnalisées » (Figure $4 A$ droite) utilisables notamment pour détecter la présence de cytotoxines [18]. Ces mesures par SPR semblent compliquées par le fait que le signal obtenu est faible, mais Liu et Chang [18] démontrent la possibilité d'amplifier ce signal. Afin de permettre l'incorporation fonctionnelle des protéines dans la bicouche, un second type d'architecture a été développé, notamment par l'équipe de Sackmann [16], et consiste à insérer un espace entre la bicouche et le support solide. Cet espace est obtenu par le dépôt d'un polymère poreux (Figure 4B) ou par l'incorporation dans la membrane de lipides fonctionnalisés, composés d'une ancre lipidique insérée dans la bicouche, d'un pont de longueur connue assurant l'espace entre la membrane et la surface solide, et d'un domaine directement fixé au support (Figure $A B$ centre) [19]. Cette construction sur coussin possède l'avantage d'accroître la fluidité de la membrane lipidique et, de ce fait, améliore le biomimétisme. Le support solide peut être choisi afin de permettre la détection de molécules passant à travers les protéines membranaires et s'accumulant sous la bicouche. Ce système permet ainsi de détecter le fonctionnement de pores intégrés dans la membrane biomimétique. Terretaz et al. [20] ont ainsi développé un pore chimérique comprenant un pore ionique et un domaine récepteur modulable. La partie réceptrice est NANP, l'épitope majeur de la protéine circumsporozoïte de Plasmodium falciparum, l'agent responsable du paludisme. Les auteurs ont montré que l'on pouvait observer, par mesure d'impédance, la modulation de l'activité du pore ionique en fonction de la fixation d'anticorps sur la partie réceptrice et, ainsi, détecter la présence d'anticorps dans le sérum (Figure $4 B$ droite). La détection directe dans le sérum est une condition favorable pour l'émergence de capteurs pour la médecine personnalisée ou un suivi peu contraignant des patients. De plus, la technologie de mesure d'impédance utilisée est miniaturisable et compatible avec l'émergence de biocapteurs implantables pour le suivi des maladies chroniques à forts risques de récidives, voire d'évolution (métastases). Un autre exemple utilisant cette architecture concerne la famille des GPCR. Cette famille de protéines est particulièrement importante pour l'industrie pharmaceutique puisqu'elles représentent à elles seules la majorité des protéines membranaires constituant des cibles thérapeutiques pour les médicaments actuels ${ }^{1}$. La famille des GPCR comprend les récepteurs cellulaires des hormones et des neurotransmetteurs, ce qui lui donne un rôle essentiel dans la communication cellulaire. Elle comprend également les récepteurs sensoriels responsables de la vue et de l'olfaction. Une méthode utilisant la résonance plasmonique de surface à partir d'une bicouche lipidique dans laquelle est insérée la rhodopsine a été développée et permet d'observer l'activation de cette protéine [21]. Une telle méthode appliquée à toutes les GPCR permettrait non seulement de détecter les ligands connus et d'intérêt diagnostique, mais également de réaliser des criblages pour de nouveaux ligands à potentiel thérapeutique.

Enfin, plusieurs laboratoires ont développé l'utilisation de ces membranes biomimétiques incorporant des protéines membranaires non plus seulement pour la détection d'une fonction, mais pour le transport spécifique d'ions ou de molécules de part et d'autre de la membrane. Dans ce cas, l'architecture sur support solide est inappropriée. En effet, deux vrais compartiments sont nécessaires afin que la molécule sélectionnée ne se retrouve pas rapidement en concentration excessive dans le compartiment accepteur. C'est pourquoi une troisième architecture (Figure $4 C$ ) a été développée, dans laquelle la membrane se retrouve en suspension dans

Voir à ce sujet le numéro thématique consacré par $\mathrm{m} / \mathrm{s}$ à cette famille GPCR (récepteurs couplés aux protéines $\mathrm{G}$ ) en octobre 2012 ( $n^{\circ} 10$, vol. 28) 




Figure 4. Architecture des membranes biomimétiques. A. Architecture de base avec exemple de capteurs pour la détection d'interactions lipides-protéines ou protéines-protéines. B. Architecture sur coussin de polymères ou espaceurs avec exemple d'un capteur de détection d'anticorps dans le sérum. C. Architecture en support poreux avec exemple d'une pile solaire à protons et d'un filtre de désalinisation de l'eau par filtration.

L'aquaporine est, de plus, une des rares protéines membranaires dont la production est aisée et se fait directement à partir du sang de différents animaux [24, 25] Elle permet de faire passer sélectivement les molécules d'eau à travers la membrane sans laisser passer les sels [26] (Figure 4C droite). Aujourd'hui une société nommée Aquaporin (http://www. aquaporin.dk) commercialise ce type de filtres. Selon le même modèle, I'utilisation des protéines membranaires impliquées dans le transport d'ions représente un réel intérêt pour la récupération d'éléments rares ou polluants

un support percé de nanopores. Cette architecture a permis d'utiliser la bactériorhodopsine (BR) [22], qui est une protéine de la famille des halobactéries transformant l'énergie solaire en un gradient de protons (Figure $4 C$ centre). Dans cette architecture poreuse, la bactériorhodopsine est enchâssée dans une membrane lipidique incluse dans les pores et permet, sous l'effet de la lumière, l'accumulation de protons dans le compartiment inférieur. Le potentiel chimique lié au gradient de protons peut alors être utilisé pour produire un courant électrique grâce à des phénomènes d'oxydoréduction au niveau des électrodes. Cette architecture permettant la séparation entre deux compartiments est également attrayante dans le domaine de la filtration, en particulier en utilisant l'aquaporine. C'est en effet une protéine dont la fonction physiologique, découverte par l'équipe de P. Agre [23], est d'accélérer le phénomène de diffusion de l'eau à travers la membrane sous l'effet de la pression osmotique [28] $(\rightarrow)$. $(\rightarrow)$ Voir la Synthèse de S. Gravelle et al., $\mathrm{m} / \mathrm{s} \mathrm{n}{ }^{\circ} 2$, février 2015 , page 174 (métaux lourds) à partir d'eau souillée. Le domaine des membranes biomimétiques représente ainsi une source importante d'innovation impactant principalement les méthodes de diagnostic et la séparation, voire l'extraction sélective, d'éléments.

\section{Méthodes de mesures actuelles et apports des capteurs biomimétiques}

Le capteur de glucose implantable permet aujourd'hui d'avoir un recul de quelques années sur l'impact réel de ces outils sur la santé et le bien-être du patient. L'étude de Raccah et al. [27] permet ainsi d'observer un vrai gain en termes d'optimisation de la gestion du diabète de type 1 puisque cela permet de mieux contrôler les constantes glycémiques au fil du temps, 
même par comparaison avec les patients munis d'une pompe automatique à insuline. Ces outils possèdent donc des avantages particulièrement bénéfiques dans le cas de pathologies nécessitant un suivi régulier ou un diagnostic le plus précoce possible. II est important de noter que, si les effets bénéfiques sont avérés pour un système implantable, ils sont déjà présents pour un système permettant au patient de réaliser un autodiagnostic. Ainsi, le développement des outils permettant aux patients de contrôler leur glycémie plusieurs fois par jour a été une avancée primordiale pour la gestion de leur diabète. Le développement des capteurs biomimétiques peut donc être étagé en deux étapes successives visant l'autodiagnostic puis l'automatisation.

Outre le diabète, de nombreuses autres maladies ou constantes biologiques pourraient bénéficier des avantages d'un contrôle par système biomimétique afin de gérer plus efficacement la santé des patients. Par exemple lors d'infections bactériennes, le diagnostic et le suivi sont assurés par des méthodes de culture, de détection par anticorps (ELISA, agglutination), de PCR, etc., qui nécessitent un laboratoire d'analyse. Le développement de capteurs biomimétiques capables de détecter directement les marqueurs de l'infection serait donc un avantage pour son suivi. De tels capteurs offriraient une aide importante dans la gestion de l'apparition de résistances aux antibiotiques puisque la reprise infectieuse pourrait rapidement être observée. II en est de même pour le suivi des patients au décours d'un traitement anti-cancéreux afin de pouvoir détecter précocement une récidive ou une dissémination de la maladie. $\diamond$

\section{SUMMARY}

\section{Biomimetic sensors in biomedical research}

The recent research on both the synthesis of membrane proteins by cellfree systems and the reconstruction of planar lipid membranes, has led to the development of a cross-technology to produce biosensors or filters. Numerous biomimetic membranes are currently being standardized and used by the industry, such as filters containing aquaporin for water desalination, or used in routine at the laboratory scale, for example the bacteriorhodopsin as a light sensor. In the medical area, several fields of application of these biomimetic membranes are under consideration today, particularly for the screening of therapeutic molecules and for the developing of new tools in diagnosis, patient monitoring and personalized medicine. $\diamond$

\section{LIENS D'INTÉRÊT}

Les auteurs déclarent n'avoir aucun lien d'intérêt concernant les données publiées dans cet article.

\section{REMERCIEMENTS}

Les auteurs remercient Marie-Claire Dagher pour son aide éditoriale.

\section{RÉFÉRENCES}

1. Kirsch J., Siltanen C., Zhou $\varrho$, et al. Biosensor technology: recent advances in threat agent detection and medicine. Chem Soc Rev 2013 ; 42 : 8733-68.

2. Petschnigg J, Moe OW, Stagljar I. Using yeast as a model to study membrane proteins. Curr Opin Nephrol Hypertens $2011 ; 4: 425-32$.
3. Krogh A, Larsson B, Von Heijne G, Sonnhammer ELL. Predicting transmembrane protein topology with a hidden Markov model: application to complete genomes. J Mol Biol 2001 ; 305 : 567-80.

4. Schlegel S, Hjelm A, Baumgarten T, et al. Bacterial-based membrane protein production. Biochim Biophys Acta 2014 ; 1843 : 1739-49.

5. Renthal R. Helix insertion into bilayers and the evolution of membrane proteins. Cell Mol Life Sci 2010 ; 67 : 1077-88.

6. Miroux B, Walker JE. Over-production of proteins in Escherichia coli: mutant hosts that allow synthesis of some membrane proteins and globular proteins at high levels. J Mol Biol 1996 ; 260 : 289-98.

7. Valderrama-Rincon JD, Fisher AC, Merritt JH, et al. An engineered eukaryotic protein glycosylation pathway in Escherichia coli. Nat Chem Biol $2012 ; 8$ : 434-36.

8. Torrent M, Llompart B, Lasserre-Ramassamy $S$, et al. Eukaryotic protein production in designed storage organelles. BMC Biol 2009; $7: 5$.

9. Dvir H, Choe S. Bacterial expression of a eukaryotic membrane protein in fusion to various mistic orthologs. Protein Expr Purif 2009; $68: 28-33$.

10. Isaksson L, Enberg J, Neutze R, et al. Expression screening of membrane proteins with cell-free protein synthesis. Protein Expr Purif $2012 ; 82$ : 218-25.

11. Deniaud A, Liguori L, Blesneac I, et al. Crystallization of the membrane protein hVDACl produced in cell-free system. Biochim Biophys Acta 2010; $1798: 1540-6$.

12. Klammt C, Schwarz D, Fendler K, et al. Evaluation of detergents for the soluble expression of $\alpha$-helical and $\beta$-barrel-type integral membrane proteins by a preparative scale individual cell-free expression system. FEBS J 2005 ; 272 : 6024-38.

13. Wada T, Shimono K, Kikukawa T, et al. Crystal structure of the eukaryotic light-driven proton-pumping rhodopsin, Acetabularia rhodopsin II, from marine alga. J Mol Biol 2011 ; 411 : 986-98.

14. Liguori L, Marques B, Villegas-Mendez A, et al. Liposomes-mediated delivery of pro-apoptotic therapeutic membrane proteins. J Control Release 2008 ; $126: 217-27$.

15. Lenormand JL. Formation of proteoliposomes containing membrane proteins by means of an acellular protein synthesis system. Patent US20100189774, 2008.

16. Sackmann $\varepsilon$. Supported membranes: scientific and practical applications. Science $1996 ; 271: 43-8$.

17. Wang Z, Wilkop T, Han JH, et al. Development of air-stable, supported membrane arrays with photolithography for study of phosphoinositideprotein interactions using surface plasmon resonance imaging. Anal Chem $2008 ; 80: 6397-404$

18. Liu Y. and Cheng Q. Detection of membrane-binding proteins by surface plasmon resonance with an all-aqueous amplification scheme. Anal Chem $2012 ; 84: 3179-86$.

19. Case GD, Worley JF. Capteur à membrane mince comprenant un interrupteur biochimique. Brevet W01993010212 Al, 1992.

20. Terrettaz S, Follonier S, Makohliso S, Vogel H. A synthetic membrane protein in tethered lipid bilayers for immunosensing in whole blood. J Struct Biol $2009 ; 168: 177-82$.

21. Bieri C, Ernst OP, Heyse $S$, et al. Micropatterned immobilization of a $G$ protein-coupled receptor and direct detection of $G$ protein activation. Nat Biotech $1999 ; 17$ : 1105-8.

22. Saeedi P, Mohammadian Moosaabadi J, Sina Sebtahmadi S, et al. Potential applications of bacteriorhodopsin mutants. Bioengineered $2012 ; 3: 326-8$.

23. Preston GM, Piazza Carroll T, Guggino WB, Agre P. Appearance of water channels in Xenopus oocytes expressing red cell CHIP28 protein. Science $1992 ; 256: 385-7$.

24. Murata K, Mitsuoka K, Hirai T, et al. Structural determinants of water permeation through aquaporin-1. Nature $2000 ; 407: 599-605$.

25. Sui H, Han BG, Lee JK, et al. Structural basis of water-specific transport through the APPI water channel. Nature $2001 ; 414: 872-8$.

26. Tang C, Qiu C, Zhao $Y$, et al. Membranes composites en film mince à base d'aquaporine. Brevet W02013043118 Al, 2012.

27. Raccah D, Sulmont V, Reznik Y, et al. Incremental value of continuous glucose monitoring when starting pump therapy in patients with poorly controlled type 1 diabetes. Diabetes Care 2009 ; 32 : 2245-9.

28. Gravelle S, Joly L, Detcheverry F, et al. Perméabilité optimale des aquaporines : une histoire de forme? Med Sci (Paris) $2015 ; 31$ : 174-9.

\section{TIRÉS À PART}

J.L. Lenormand 